\title{
A dual but asymmetric role of the dorsal anterior cingulate cortex in response inhibition and switching from a non-salient to salient action
}

Peter Manza ${ }^{1,2, *}$, Sien $\mathrm{Hu}^{1, *}$, Herta H. Chao ${ }^{3,4}$, Sheng Zhang ${ }^{1}$, Hoi-Chung Leung ${ }^{2}$, Chiang-shan R. $\mathrm{Li}^{1,5,6}$

${ }^{1}$ Department of Psychiatry, Yale University, New Haven, CT 06519

${ }^{2}$ Department of Psychology, Stony Brook University, Stony Brook, NY 11790

${ }^{3}$ Department of Internal Medicine, Yale University New Haven, CT 06519

${ }^{4}$ Medical Service, VA Connecticut Health Care System, West Haven, CT 06516

${ }^{5}$ Department of Neuroscience, Yale University, New Haven, CT 06520

${ }^{6}$ Interdepartmental Neuroscience Program, Yale University, New Haven, CT 06520

(Running title: dACC, switching and inhibition)

*PM and SH contributed equally to the current work.

Address correspondence to:

Dr. C.-S. Ray Li

Connecticut Mental Health Center S112

34 Park Street

New Haven CT 06519

Phone: 203-974-7354

FAX: 203-974-7076

Email: chiang-shan.li@yale.edu 


\begin{abstract}
Response inhibition and salience detection are among the most studied psychological constructs of cognitive control. Despite a growing body of work, how inhibition and salience processing interact and engage regional brain activations remains unclear. Here, we examined this issue in a stop signal task (SST), where a prepotent response needs to be inhibited to allow an alternative, less dominant response. Sixteen adult individuals performed two versions of the SST each with 25\% (SST25) and 75\% (SST75) of stop trials. We posited that greater regional activations to the infrequent trial type in each condition (i.e., to stop as compared to go trials in SST25 and to go as compared to stop trials in SST75) support salience detection. Further, successful inhibition in stop trials requires attention to the stop signal to trigger motor inhibition, and the stop signal reaction time (SSRT) has been used to index the efficiency of motor response inhibition. Therefore, greater regional activations to stop as compared to go success trials in association with the stop signal reaction time (SSRT) serves to expedite response inhibition. In support of an interactive role, the dorsal anterior cingulate cortex (dACC) increases activation to salience detection in both SST25 and SST75, but only mediates response inhibition in SST75. Thus, infrequency response in the dACC supports motor inhibition only when stopping has become a routine. In contrast, although the evidence is less robust, the pre-supplementary motor area (pre-SMA) increases activity to the infrequent stimulus and supports inhibition in both SST25 and SST75. These findings clarify a unique role of the dACC and add to the literature that distinguishes dACC and pre-SMA functions in cognitive control.
\end{abstract}

Key words: conflict; inhibitory control; task switching; fMRI, dorsomedial prefrontal cortex 


\section{Introduction}

Response inhibition, the ability to rapidly cancel an action, is a critical executive function. The stop-signal task (SST) is widely used to study response inhibition, where individuals respond to the same stimulus repeatedly and on a minority of trials must cancel this prepotent response (Logan et al., 1984). Previous functional magnetic resonance imaging (fMRI) studies using the SST have identified a network of brain regions that include the inferior frontal gyrus, insula, dorsomedial prefrontal cortex, and the basal ganglia, in response to stop versus go trials (see Li, 2014 for a review). However, whether these regions are specifically involved in motor inhibition, rather than saliency response, as in switching from a frequent to an infrequent action, remains unclear (Kenner et al., 2010; Obeso et al., 2013).

Previous studies have attempted to dissociate response-switching, or the ability to adapt to changing task demands, (Badre and Wagner, 2006; Jurado and Rosselli, 2007; Leber et al., 2008) and response inhibition, by altering the SST to include trials where subjects switch from one type of response to another, in addition to trials that require the dominant response to be stopped. However, these attempts yielded varied results. Kenner et al. (2010) reported that many cortical regions activated to both stop and switch trials, suggesting a general role in switching as opposed to inhibition. On the other hand, transcranial magnetic stimulation (TMS) of the presupplementary motor area (pre-SMA) disrupted stopping, but not switching (Obeso et al., 2013). Further, Roberts and Husain (2015) recently reported that an individual with a lesion to the caudal pre-SMA showed impairment in switching but not stopping. While these discrepancies may reflect differences in methodology (e.g., TMS vs. lesion; variation in brain locations examined), a common limitation is that both stop and switch trials are presented infrequently. Therefore, both trial types confound salient stimulus detection (responses to infrequent, arousing events; Horvitz, 2000) with the behavior of interest and require a form of "switching” from a dominant to a non-dominant action plan.

In the response-switching literature, efforts have been made to understand the role of salience detection by manipulating whether a task shift requires alternation from a dominant to a non-dominant action. In these reports, shifts to an infrequent stimulus type, compared to a frequent stimulus type, engage a wide array of cortical and subcortical structures (Badre and Wagner, 2006; Braver et al., 2001; De Baene and Brass, 2013; Dove et al., 2000; Rushworth et al., 2001). On the other hand, the role of inhibition in these processes remains unclear, as 
response switching engages inhibition of the current, prevailing response (Koch et al., 2010; Wessel and Aron, 2013) and yet most studies do not provide a quantifiable measure of motor inhibition. Thus, it remains a challenge to distinguish activities related to rule switch (as in alternating between two equiprobable actions) or saliency (as in shifting from a frequent to less frequent response) from those related to motor inhibition.

Here, to address this issue, we used a within-subject design where 16 individuals performed two variants of the SST during fMRI: a conventional version with 25\% stop and 75\% go trials (SST25), and a modified version (SST75) where trial probabilities were reversed with $75 \%$ stop and 25\% go trials. While in SST25 a successful stop, as compared to go trial, involves both response inhibition and detection of a salient (infrequent) stimulus, in SST75 the same contrast involves response inhibition but not salience detection as stop trials dominate the task. Using this design, we hoped to dissociate regional activations to response inhibition and saliency processing. In particular, in the SST, we can obtain a measure of stop signal reaction time (SSRT) to quantify the efficiency of response inhibition (Logan et al., 1984). A shorter SSRT indicates a more efficient process of response inhibition. Thus, greater regional activations to stop as compared to go success trials in association with the SSRT serves to expedite response inhibition (Chao et al., 2009; Zhang et al., 2015). Greater regional activations both to stop as compared to go trials in SST25 and to go as compared to stop trials in SST75 support salience detection. We examined neural processes both shared by and distinct to saliency processing and motor inhibition.

\section{Methods and Materials}

The study was performed under protocols approved by the Yale Human Investigation and MRI Safety Committees. Sixteen adults ( 8 females, mean age of $29 \pm 6$ years) participated in the experiment. All participants were free from medical, neurological and psychiatric illnesses, denied use of illicit substances and tested negative in urine screen on the day of fMRI.

\section{Behavioral Task}

During fMRI, participants performed the stop-signal task (SST; Logan et al., 1984; Fig. 1A) as in our previous work (Farr et al., 2012; Hu et al., 2014; Winkler et al., 2013). There were two trial types: go and stop, randomly intermixed. A small dot appeared on the screen to engage 
attention at the beginning of a go trial. After a randomized time interval (fore-period) between 1 and $5 \mathrm{~s}$, the dot turned into a circle (the go signal), prompting the subject to quickly press a button. The circle vanished at a button press or after $1 \mathrm{~s}$ had elapsed, whichever came first, and the trial terminated. A premature button press prior to the appearance of the circle also terminated the trial. In a stop trial, an additional X, the stop signal, appeared after and replaced the go signal. The subjects were told to withhold their button press upon seeing the stop signal. The stop-signal delay (SSD) — the time interval between the go and stop signal — started at 200 ms and varied from one stop trial to the next according to a staircase procedure, increasing and decreasing by 67 ms each after a successful and failed stop trial (De Jong et al., 1990; Levitt, 1971). There was an intertrial interval of $2 \mathrm{~s}$. Subjects were instructed to respond to the go signal quickly while keeping in mind that a stop signal could come up in a small number of trials. Depending on the actual stimulus timing (trials varied in fore-period duration) and speed of response, the total number of trials varied slightly across subjects in an experiment. With the staircase procedure, we anticipated that the subjects would succeed in withholding their response in approximately half of the stop trials. The stop-signal reaction time (SSRT) was computed by subtracting the critical stop-signal delay, or the estimated SSD required for a subject to get half of stop trials correct, from the median go RT (Li et al., 2008).

We computed post-error slowing (PES) as described in detail in our earlier studies (Ide and Li, 2011; Ide et al., 2015; Li et al., 2006; Liao et al., 2014). Briefly, four main types of trial outcome were first distinguished: go success (GS), go error (F), stop success (SS), and stop error (SE) trial. GS trials were divided into those that followed a GS (pGS), F (pF), SS (pSS), and SE (pSE) trial. The effect size of a two-sample t test on the RT of pSE and pG trials was used to index PES for individual subjects. We also computed the "fore-period effect" (Li et al., 2005). For each subject, the trials were divided into two groups, one with a fore-period of less than $3 \mathrm{~s}$ (the approximate mean duration of the fore-period) and the other with one equal to or longer than 3 s. Fore-period effect $=$ mean RT for short fore-period $(<3 \mathrm{~s})-$ mean RT for long fore-period $(\geq 3 \mathrm{~s}$ ). Likewise, a $\mathrm{t}$ test was performed on the RT of short and long fore-periods and the effect size was used as an index of the fore-period effect for each individual subject.

Participants performed two different versions of the SST during fMRI. In one version, approximately three quarters of all trials were go trials, and the remaining one quarter were stop trials (SST25). In the alternate version, trial probabilities were reversed: approximately three 
quarters of all trials were stop trials, and the remaining one quarter were go trials (SST75). Figure 1B summarizes the constructs measured in SST25 and SST75. Every participant completed three 8-minute sessions each of SST25 and SST75 during the same scan, with the order counterbalanced across subjects.

\section{Imaging protocol and spatial preprocessing of brain images}

Conventional T1-weighted spin-echo sagittal anatomical images were acquired for slice localization using a 3T scanner (Siemens Trio). Anatomical images of the functional slice locations were obtained with spin-echo imaging in the axial plan parallel to the Anterior Commissure-Posterior Commissure (AC-PC) line with TR=300 ms, TE=2.5 ms, bandwidth=300 $\mathrm{Hz} /$ pixel, flip angle $=60^{\circ}$, field of view $=220 \times 220 \mathrm{~mm}$, matrix $=256 \times 256$, 32 slices with slice thickness=4 mm and no gap. A single high-resolution T1-weighted gradient-echo scan was obtained. One hundred and seventy-six slices parallel to the AC-PC line covering the whole brain were acquired with $\mathrm{TR}=2530 \mathrm{~ms}$, TE=3.66ms, bandwidth $=181 \mathrm{~Hz} /$ pixel, flip angle $=7^{\circ}$, field of view $=256 \times 256 \mathrm{~mm}$, matrix $=256 \times 256,1 \mathrm{~mm}^{3}$ isotropic voxels. Functional blood oxygenation level dependent (BOLD) signals were then acquired with a single-shot gradientecho echo-planar imaging (EPI) sequence. Thirty-two axial slices parallel to the AC-PC line covering the whole brain were acquired with TR=2000 ms, TE=25 ms, bandwidth=2004 $\mathrm{Hz} /$ pixel, flip angle $=85^{\circ}$, field of view $=220 \times 220 \mathrm{~mm}$, matrix $=64 \times 64$, 32 slices with slice thickness $=4 \mathrm{~mm}$ and no gap. There were thus 240 images in each session for a total of 6 sessions.

Data were analyzed with Statistical Parametric Mapping (SPM8, Wellcome Department of Imaging Neuroscience, University College London, U.K.). In the pre-processing of BOLD data, images of each participant were realigned (motion-corrected) and corrected for slice timing. A mean functional image volume was constructed for each participant for each run from the realigned image volumes. These mean images were co-registered with the high resolution structural image and then segmented for normalization to an MNI (Montreal Neurological Institute) EPI template with affine registration followed by nonlinear transformation (Ashburner and Friston, 1999; Friston et al., 1995). Finally, images were smoothed with a Gaussian kernel of $8 \mathrm{~mm}$ at Full Width at Half Maximum. Images from the first five TRs at the beginning of each trial were discarded to enable the signal to achieve steady-state equilibrium between radio frequency pulsing and relaxation. 


\section{Generalized linear models}

Our goal was to identify regional activations in the SST25 and SST75 that might support response inhibition vs. salience detection. To this end, a statistical analytical design was constructed for each individual subject, using the general linear model (GLM) with the onsets of go signal in each of the four main trial types (GS, F, SS, SE) convolved with a canonical hemodynamic response function (HRF) and with the temporal derivative of the canonical HRF and entered as regressors in the model (Friston et al., 1995). Realignment parameters in all 6 dimensions were also entered in the model. Serial autocorrelation was corrected by a first-degree autoregressive or AR(1) model. The GLM estimated the component of variance that could be explained by each of the regressors. In the first-level analysis, we constructed for each individual subject a contrast between SS and GS (SS > GS). The contrast (difference in $\beta$ ) images of the first-level analysis were then used for the second-level group statistics (random effect analysis; Penny and Holmes, 2003). In the second level analysis, all images were evaluated at a voxelwise threshold of $p<.005$, combined with a cluster size threshold of 29 contiguous voxels $\left(783 \mathrm{~mm}^{3}\right)$. This combined threshold was estimated with a Monte-Carlo simulation using AlphaSim (Douglas Ward, http://afni.nimh.nih.gov/pub/dist/doc/program_help/AlphaSim.html) to give an overall threshold of $p<.05$, corrected for multiple comparisons across the entire brain.

One-sample t tests were performed on the SS > GS contrast to obtain the group effects each for SST25 and SST75. To examine if SST25 and SST75 shared regional activations during stopping, we performed a conjunction analysis on SS > GS in SST25 and SST75, using the minimum statistic compared to the conjunction null method (Nichols et al., 2005) implemented in SPM8. Further, to examine if any regions showed common activation to infrequent trial type (salience detection) in both SST25 and SST75, we performed a conjunction analysis on SS > GS in SST25 and GS > SS in SST75.

The conjunction analyses identified regional activations to stopping and salience detection that were shared in SST25 and SST75. On the other hand, salience detection essentially involves enhanced attention to infrequency and/or diminished planning for the prepotent response and these preparatory processes may be represented differently and influence inhibitory control differently between SST25 and SST75 (Cai et al., 2014; Hu and Li, 2012; Zhang et al., 2015). Thus, we performed paired t tests to identify differences in voxelwise responses to SS > 
GS in SST25 vs. SST75. Greater activation during SS > GS in SST25 than SST75 would identify the salience-related activities that may not be equally represented in the two conditions and eluded conjunction analysis as a result. In a region of interest analysis, we then queried how these differences in regional activations related to inhibitory control by performing a linear regression against SSRT for each experimental condition.

\section{Results}

Table 1 summarizes the behavioral results, and Figure 2a shows the inhibitory function of individual subjects in each condition. Individuals responded significantly more slowly on go trials and on stop error trials in SST75, as compared to SST25. However, the SSRT did not significantly differ between the two conditions.

For regional activations to stopping, the results of one-sample t-tests for SS > GS are shown in Figure 2b. In SST25, significant activations were found in bilateral superior/middle/inferior frontal gyri (IFG), bilateral insulae, dorsomedial frontal cortex (dmFC) including dorsal anterior cingulate cortex (dACC) and pre-supplementary motor area (pre-SMA), bilateral inferior parietal lobule (IPL), and bilateral visual cortex. In SST75, significant activations were only observed in bilateral visual cortex, left orbitofrontal cortex, and right precentral gyrus (PrCG). The results of one-sample t-tests for GS > SS are shown in Figure 2c. In SST25, significant activations were found in cuneus/superior parietal gyrus. In SST75, significant activations were observed in cuneus/parieto-occipital fissure/superior parietal gyrus, as well as in the left insula, thalamus, midbrain, cerebellum, dACC, and left PrCG and postcentral gyrus (PoCG).

In conjunction analysis for stopping, bilateral visual cortices respond to SS $>$ GS in both SST25 and SST75 (Figure 3a). This activity of the visual cortex was not significantly correlated with SSRT in SST25 or SST75 (p's > 0.10; Pearson regression; Figure 3b). In conjunction analysis for salience detection, we identified a small cluster in the dACC (center coordinates at [3 29 25]) that increased activation to SS > GS in SST25 and to GS > SS in SST75 (Figure 3c). Further, the effect size of dACC activity during SS > GS correlated negatively to SSRT in SST75 ( $p=0.02$, Pearson regression) but not in SST25 ( $p=0.28$; Figure 3d). The latter finding suggests that this dACC cluster not only plays a role in detecting salient, infrequent stimuli but also expedites response inhibition when stop trials dominate the task. 
We performed paired-sample t-tests on the brain activations for the contrast SS > GS (Figure 2d, Table 2). In SST25, as compared to SST75, activations were higher in a number of regions including large clusters in bilateral IFG and insulae that extended into striatum and thalamus, midbrain, dmFC including dACC and pre-SMA, left middle temporal gyrus (MTG), right ITG, dorsal cerebellum and cerebellar vermis. No regions showed significantly higher activation in SST75 as compared to SST25.

To examine whether these regional activities are related to inhibitory control, we performed a voxelwise linear regression against SSRT each in SST25 and SST75, masked by the combined clusters shown in Figure 2d and Table 2 (i.e., the clusters from the SS > GS [SST25 > SST75]). The results showed that, in SST25, no voxel activities were correlated significantly to SSRT. In SST75, clusters at the dACC, pre-SMA and thalamus/putamen showed an effect size that correlated negatively to SSRT (Figure 4a; Table 3). Figure 4b shows the effect size of SS $>$ GS for individual regions. We then determined if the slopes of the regressions were significantly different between the two conditions (Zar, 2010). Of these three clusters showing a

significant correlation in SST75, the dACC in a nearly identical location (center coordinates at [9 26 37]) as identified from conjunction analysis showed a significant difference in slope between SST25 and SST75: (SST25, $\mathrm{r}=-.02$; SST75, $\mathrm{r}=-.69 ; \mathrm{z}=-2.11, p=.035)$. On the other hand, the anterior pre-SMA showed a significant negative correlation during SS $>$ GS in SST75 ( $\mathrm{r}=-.59, p$ $=.02$ ) and a trend toward significant negative correlation during SS $>$ GS in SST25 ( $\mathrm{r}=-.48$; $p=.06)$. Figure $4 c$ plots the linear regression for these regions.

\section{Discussion}

By making the stop and go trial an infrequent condition each in two versions of the SST, we examined whether previously reported regional activations to response inhibition are related to stopping or more generally to detection of salient, infrequent stimuli. By correlating these activities with SSRT, we confirmed a role in motor response inhibition for these regions.

Behaviorally, participants responded more slowly and made more commission errors on go trials in SST75 than SST25, due to the higher frequency of stop trials in SST75. This replicates previous findings of increasing RT with higher proportions of stop trials in the SST or no-go trials in the Go-NoGo paradigm (Wijeakumar et al., 2015; Zandbelt and Vink, 2010). However, SSRT did not significantly differ between the two experimental conditions, suggesting 
that whether or not stopping was the more frequent response did not alter the efficiency of response inhibition, again replicating previous work (Vink et al., 2015; Zandbelt et al., 2013).

\section{Cognitive control networks: stopping vs. salience detection}

Previous studies have attempted to determine whether brain regions within the cognitive control network support true stopping behaviors, rather than more generally alternating between action plans. However, there have been inconsistent findings, perhaps because in those studies, stop and switch trials are both presented infrequently, thus confounding salience detection with the construct of interest (Kenner et al., 2010; Obeso et al., 2013). To avoid this potential issue, we manipulated stop trial frequency across two versions of the SST so that we could assess brain responses to stop trials when they represent the dominant or non-dominant action plan. Our results largely support the notion that some regions typically implicated in stopping, such as the IFG, respond primarily to salient or infrequent stimuli (Hampshire and Sharp, 2015; Hampshire et al., 2010). These and several other areas including the cerebellum, thalamus, and striatum were most strongly activated to the infrequent trial type, and not specifically to stopping. This is consistent with an extensive literature showing IFG response to salient stimuli even when motor

inhibition is not required (Hampshire and Sharp, 2015; Hampshire, 2015; Hampshire et al., 2010, 2009; Walther et al., 2011) and without differentiating successful and unsuccessful stops (Cai et al., 2014). Our data also showed that the thalamus and striatum play a similar role during SST performance. These results are very similar to a recent study that manipulated trial probability in a Go/NoGo task (Wijeakumar et al., 2015), supporting a role of the thalamus and striatum in salience detection across different behavioral tasks.

Direct evidence for how saliency affects neural processing during response inhibition, however, remains limited. Boehler et al. (2011) modified the SST so that in one version of the task, stop signals appeared but were irrelevant. In this condition, subjects were instructed to simply respond to all go stimuli and ignore any stop signals. They found that compared to stopirrelevant trials, stop-relevant versus go trials elicited stronger activation in pre-SMA, IPL, right IFG/insula, and right middle frontal gyrus, suggesting that these regions facilitate response inhibition when stop trials are more salient. Yet, as we have elaborated in our previous work, "stopping" in the SST involves multiple processes including proactive control, attention to the stop signal and motor inhibition triggered by the stop signal (Duann et al., 2009; Hu et al., 2015). 
Thus, a contrast of stop versus go success trials does not serve to isolate the neural processes of motor inhibition. We proposed that a better construct to index the efficiency of response inhibition is the stop signal reaction time (SSRT). In particular, we showed that individual variation in the SSRT is reflected in the activity of the anterior pre-SMA during stopping (Li et al., 2006). Here, among all brain regions that respond to salience detection, as identified from the paired sample $t$ test, only the pre-SMA exhibited activity in negative correlation to SSRT in both SST25 and SST75, suggesting that pre-SMA mediates response inhibition in addition to salience detection. The other two clusters, dACC and thalamus/striatum, showed activity in negative correlation to SSRT in SST75 but not SST25, with the dACC showing a significant difference in slope between the two conditions. The latter finding suggests an interactive role of the dACC in salience detection and inhibition, as also shown by the conjunction analysis.

\section{A dual but asymmetric role of the dACC in response inhibition and salience detection}

The dACC responded to the infrequent trial type in a Go/No-Go task where trial type probabilities were reversed (Braver et al., 2001). Yet in disjunction analysis, a right-lateralized portion of the dACC with a location similar to what we identified here was specifically active when the infrequent trial type required a "No-Go" response. Thus, while dACC detects infrequent stimuli, at least part of the dACC may also have a more specific role in response inhibition. Here, we found that in SST75, higher stop relative to go activations in the dACC correlated with faster SSRT. Therefore, while the dACC was overall more responsive to the infrequent trial type, higher dACC activation to the frequent stop trials and/or lower dACC activation to the infrequent go trials predicted better response inhibition performance. That is, the saliency activity of the dACC is relevant to the efficiency of response inhibition only when stopping is a routine - when the action of stopping is no longer salient. This contrasts with the pre-SMA, where saliency response is in link with efficient response inhibition whether stopping is salient or not, in broad support of its role in inhibitory control (Hirose et al., 2012; Konishi et al., 2010; Sharp et al., 2010; Watanabe et al., 2015).

In line with this finding, Garavan et al. (2002) categorized stop trials as being “easy” if the RT was long on the preceding go trial, hypothesizing that recent response slowing reflects proactive control and facilitates response inhibition. They found that easy response inhibition was associated with lower dACC activity to the infrequent trial type. That is, when participants 
were already prepared to stop, a stop trial was no longer salient and elicited little response from the dACC. These findings are broadly in accord with single-cell recordings in animals and human fMRI data suggesting that the dACC encodes a "surprise” signal, or the absolute difference between the expected and actual outcome of an event (Hayden et al., 2011; Hu et al., 2015; Ide et al., 2013). The difference in motor response requirements between SST25 and SST75 could be another factor that interacts with dACC surprise signals to produce this result. DACC activations supporting cognitive control vary with specific motor responses and task designs (Liston et al., 2006; Luks et al., 2002; Muhle-Karbe et al., 2014; Xu et al., 2015), and the dACC may play a role in motor output specifically for salient or infrequent events (Menon and Uddin, 2010; Uddin, 2015). Thus, higher dACC “surprise” activation predicts poorer task performance when the infrequent event requires motor output, as in SST75. Conversely, when infrequent events do not require motor output, as in SST25, no association between dACC activation and SSRT is observed. Together, these considerations highlight the versatility and complexity of dACC functions.

Although thus far we have discussed how inhibition interacts with salience detection, it is important to note that response switching cannot be completely dissociated from these processes. In fact, SSRT is thought to be a product of competition between go and stop processes based on the interactive race model (Boucher et al., 2007; Verbruggen and Logan, 2008). Hence, in addition to indexing inhibition, SSRT likely also measures some form of switching between the two response modalities, as supported by experimental data (Emeric et al., 2007; Kenner et al., 2010; Zhang et al., 2015). Indeed, multiple lines of evidence have emerged to suggest that the dorsomedial frontal cortex, including the dACC and pre-SMA, play a crucial role in response switching as well as inhibition. Single-neuron recordings in monkeys show switch-related responses in this region (Isoda and Hikosaka, 2007; Stuphorn and Schall, 2006). While some human studies using TMS during the SST have concluded that the dorsomedial frontal cortex may be crucial for inhibition specifically (Chen et al., 2009; Neubert et al., 2010; Obeso et al., 2013; Watanabe et al., 2015), many have theorized that this region has a more general role in switching between action plans (Jasinska, 2013; Mostofsky and Simmonds, 2008; Nachev et al., 2008), as supported by an fMRI meta-analysis (Rae et al., 2014). Thus, while in the current study we were able to determine how salience detection influences SST performance, a true dissociation between response switching and inhibition remains to be established in future work. 


\section{Limitations and Conclusions}

The current study had several limitations. First, this study had a relatively small sample size of 16 participants, although it has been suggested that this number provides adequate power for random-effects fMRI analyses (Friston, 2012). Second, our data can only speak to cognitive control during the SST. Whereas the SST requires cancellation of an initiated response, other paradigms, such as the Go/No Go task, require action restraint, and performance on these tasks shows overlapping but also distinct neural correlates (for a meta-analysis, see Swick et al., 2011). Thus, while the current study has provided new detail on how medial frontal areas are involved in stop- and salience-related behavior during stop signal inhibition, more work is needed to precisely describe how these brain regions promote successful cognitive control more broadly. Another potential issue is the stability or temporal evolution of regional signals during the SST (Jimura et al., 2014). An earlier fMRI study engaged participants in the SST for an extended period of time (768 trials in total) and showed that regional responses to stop versus go trials are in more robust correlation with the SSRT during the later sessions of the task. Although the order of SST25 and SST75 were counter-balanced across subjects in the current work, we cannot rule out the possibility that some of the "negative" findings may result from signal instabilities in the regions of interest. This issue applies broadly to extant studies of the SST, as typically 300 trials or less were acquired in a given experiment.

To conclude, by varying the frequency of stop trials in two versions of the SST, we distinguished regional activations to stopping and salience detection. The dACC responds to salient stimuli and supports motor response inhibition, as indexed by SSRT, only when the stop trial predominates the task and is no longer salient. In contrast, the pre-SMA shows differential activity to infrequency and/or motor preparation between SST25 and SST75, and appears to expedite motor response inhibition/switching across the two conditions. 


\section{Acknowledgements:}

This study was supported by NSF grant BCS1309260 and NIH grants AA021449 and

DA023248. The funding agencies are otherwise not involved in data collection and analysis, or in the decision to publish these results. 


\section{References:}

Ashburner, J., Friston, K., 1999. Nonlinear spatial normalization using basis functions. Human brain mapping 266, 254-266.

Badre, D., Wagner, A.D., 2006. Computational and neurobiological mechanisms underlying cognitive flexibility. Proceedings of the National Academy of Sciences of the United States of America 103, 7186-7191. doi:10.1073/pnas.0509550103

Boehler, C.N., Appelbaum, L.G., Krebs, R.M., Chen, L.C., Woldorff, M.G., 2011. The role of stimulus salience and attentional capture across the neural hierarchy in a stop-signal task. PLoS ONE 6, 1-10. doi:10.1371/journal.pone.0026386

Boucher, L., Palmeri, T.J., Logan, G.D., Schall, J.D., 2007. Inhibitory control in mind and brain: an interactive race model of countermanding saccades. Psychological review 114, 376-397. doi:10.1037/0033-295X.114.2.376

Braver, T.S., Barch, D.M., Gray, J.R., Molfese, D.L., Snyder, A., 2001. Anterior cingulate cortex and response conflict: effects of frequency, inhibition and errors. Cerebral cortex 11, 825836. doi:10.1093/cercor/11.9.825

Cai, W., Ryali, S., Chen, T., Li, C.-S.R., Menon, V., 2014. Dissociable Roles of Right Inferior Frontal Cortex and Anterior Insula in Inhibitory Control: Evidence from Intrinsic and TaskRelated Functional Parcellation, Connectivity, and Response Profile Analyses across Multiple Datasets. The Journal of Neuroscience 34, 14652-14667. doi:10.1523/JNEUROSCI.3048-14.2014

Chao, H.H. a, Luo, X., Chang, J.L.K., Li, C.-S.R., 2009. Activation of the pre-supplementary motor area but not inferior prefrontal cortex in association with short stop signal reaction time--an intra-subject analysis. BMC neuroscience 10, 75. doi:10.1186/1471-2202-10-75

Chen, C.Y., Muggleton, N.G., Tzeng, O.J.L., Hung, D.L., Juan, C.H., 2009. Control of prepotent responses by the superior medial frontal cortex. NeuroImage 44, 537-545. doi:10.1016/j.neuroimage.2008.09.005

De Baene, W., Brass, M., 2013. Switch probability context (in)sensitivity within the cognitive control network. NeuroImage 77, 207-214. doi:10.1016/j.neuroimage.2013.03.057

De Jong, R., Coles, M.G., Logan, G.D., Gratton, G., 1990. In search of the point of no return: the control of response processes. Journal of experimental psychology Human perception and performance 16, 164-182. doi:10.1037/0096-1523.16.1.164

Dove, a, Pollmann, S., Schubert, T., Wiggins, C.J., von Cramon, D.Y., 2000. Prefrontal cortex activation in task switching: an event-related fMRI study. Cognitive Brain Research 9, 103109. doi:10.1016/S0926-6410(99)00029-4

Duann, J.-R., Ide, J.S., Luo, X., Li, C.R., 2009. Functional connectivity delineates distinct roles of the inferior frontal cortex and presupplementary motor area in stop signal inhibition. The Journal of neuroscience 29, 10171-9. doi:10.1523/JNEUROSCI.1300-09.2009

Emeric, E.E., Brown, J.W., Boucher, L., Carpenter, R.H.S., Hanes, D.P., Harris, R., Logan, G.D., Mashru, R.N., Paré, M., Pouget, P., Stuphorn, V., Taylor, T.L., Schall, J.D., 2007. Influence of history on saccade countermanding performance in humans and macaque monkeys. Vision Research 47, 35-49. doi:10.1016/j.visres.2006.08.032

Farr, O.M., Hu, S., Zhang, S., Li, C.S.R., 2012. Decreased saliency processing as a neural measure of Barratt impulsivity in healthy adults. NeuroImage 63, 1070-1077. doi:10.1016/j.neuroimage.2012.07.049

Friston, K., 2012. Ten ironic rules for non-statistical reviewers. NeuroImage 61, 1300-1310. doi:10.1016/j.neuroimage.2012.04.018 
Friston, K., Frith, C., Frackowiak, R., Turner, R., 1995. Characterizing dynamic brain responses with fMRI: a multivariate approach. Neuroimage 2, 166-172.

Garavan, H., Ross, T.J., Murphy, K., Roche, R. a P., Stein, E. a, 2002. Dissociable executive functions in the dynamic control of behavior: inhibition, error detection, and correction. NeuroImage 17, 1820-1829. doi:10.1006/nimg.2002.1326

Hampshire, A., 2015. Putting the brakes on inhibitory models of frontal lobe function. NeuroImage 113, 340-355. doi:10.1016/j.neuroimage.2015.03.053

Hampshire, A., Chamberlain, S.R., Monti, M.M., Duncan, J., Owen, A.M., 2010. The role of the right inferior frontal gyrus: inhibition and attentional control. NeuroImage 50, 1313-1319. doi:10.1016/j.neuroimage.2009.12.109

Hampshire, A., Sharp, D.J., 2015. Contrasting network and modular perspectives on inhibitory control. Trends in Cognitive Sciences 19, 445-452. doi:10.1016/j.tics.2015.06.006

Hampshire, A., Thompson, R., Duncan, J., Owen, A.M., 2009. Selective tuning of the right inferior frontal gyrus during target detection. Cognitive, affective \& behavioral neuroscience 9, 103-112. doi:10.3758/CABN.9.1.103

Hayden, B.Y., Heilbronner, S.R., Pearson, J.M., Platt, M.L., 2011. Surprise signals in anterior cingulate cortex: neuronal encoding of unsigned reward prediction errors driving adjustment in behavior. The Journal of neuroscience 31, 4178-4187. doi:10.1523/JNEUROSCI.465210.2011

Hirose, S., Chikazoe, J., Watanabe, T., Jimura, K., Kunimatsu, A., Abe, O., Ohtomo, K., Miyashita, Y., Konishi, S., 2012. Efficiency of Go/No-Go Task Performance Implemented in the Left Hemisphere. The Journal of Neuroscience 32, 9059-9065. doi:10.1523/JNEUROSCI.0540-12.2012

Horvitz, J., 2000. Mesolimbocortical and nigrostriatal dopamine responses to salient non-reward events. Neuroscience 96, 651-656.

Hu, S., Ide, J.S., Zhang, S., Li, C.R., 2015. Anticipating conflict: Neural correlates of a Bayesian belief and its motor consequence. NeuroImage 119, 286-295. doi:10.1016/j.neuroimage.2015.06.032

Hu, S., Li, C.-S.R., 2012. Neural processes of preparatory control for stop signal inhibition. Human brain mapping 33, 2785-96. doi:10.1002/hbm.21399

Hu, S., Tseng, Y.C., Winkler, A.D., Li, C.S.R., 2014. Neural bases of individual variation in decision time. Human Brain Mapping 35, 2531-2542. doi:10.1002/hbm.22347

Ide, J.S., Hu, S., Zhang, S., Yu, A.J., Li, C.R., 2015. Impaired Bayesian learning for cognitive control in cocaine dependence. Drug and Alcohol Dependence 151, 220-227. doi:10.1016/j.drugalcdep.2015.03.021

Ide, J.S., Li, C.S.R., 2011. A cerebellar thalamic cortical circuit for error-related cognitive control. NeuroImage 54, 455-464. doi:10.1016/j.neuroimage.2010.07.042

Ide, J.S., Shenoy, P., Yu, A.J., Li, C.R., 2013. Bayesian prediction and evaluation in the anterior cingulate cortex. The Journal of neuroscience 33, 2039-47. doi:10.1523/JNEUROSCI.2201-12.2013

Isoda, M., Hikosaka, O., 2007. Switching from automatic to controlled action by monkey medial frontal cortex. Nature neuroscience 10, 240-8. doi:10.1038/nn1830

Jasinska, A.J., 2013. Automatic inhibition and habitual control: Alternative views in neuroscience research on response inhibition and inhibitory control. Frontiers in Behavioral Neuroscience 7, 1-4. doi:http://dx.doi.org/10.3389/fnbeh.2013.00025

Jimura, K., Hirose, S., Kunimatsu, A., Ohtomo, K., Koike, Y., Konishi, S., 2014. Late 
enhancement of brain-behavior correlations during response inhibition. Neuroscience 274, 383-392. doi:10.1016/j.neuroscience.2014.05.058

Jurado, M.B., Rosselli, M., 2007. The elusive nature of executive functions: A review of our current understanding. Neuropsychology Review 17, 213-233. doi:10.1007/s11065-0079040-z

Kenner, N.M., Mumford, J. a, Hommer, R.E., Skup, M., Leibenluft, E., Poldrack, R. a, 2010. Inhibitory motor control in response stopping and response switching. The Journal of neuroscience 30, 8512-8518. doi:10.1523/JNEUROSCI.1096-10.2010

Koch, I., Gade, M., Schuch, S., Philipp, a. M., 2010. The role of inhibition in task switching: A review. Psychonomic Bulletin \& Review 17, 1-14. doi:10.3758/PBR.17.1.1

Konishi, S., Watanabe, T., Jimura, K., Chikazoe, J., Hirose, S., Kimura, H.M., Miyashita, Y., 2010. Role for presupplementary motor area in inhibition of cognitive set interference. Journal of cognitive neuroscience 23, 737-745. doi:10.1162/jocn.2010.21480 [doi]

Leber, A.B., Turk-Browne, N.B., Chun, M.M., 2008. Neural predictors of moment-to-moment fluctuations in cognitive flexibility. Proceedings of the National Academy of Sciences of the United States of America 105, 13592-13597. doi:10.1073/pnas.0805423105

Levitt, H., 1971. Transformed up-down methods in psychoacoustics. The Journal of the Acoustical Society of America 49, 467-477. doi:10.1121/1.1912375

Li, C.R., Huang, C., Constable, R.T., Sinha, R., 2006. Imaging response inhibition in a stopsignal task: neural correlates independent of signal monitoring and post-response processing. The Journal of neuroscience 26, 186-192. doi:10.1523/JNEUROSCI.374105.2006

Li, C.R., Krystal, J.H., Mathalon, D.H., 2005. Fore-period effect and stop-signal reaction time. Experimental Brain Research 167, 305-309. doi:10.1007/s00221-005-0110-2

Li, C.-S.R., 2014. Response Inhibition, in: Toga, A.W. (Ed.), Brain Mapping: An Encyclopedic Reference. Elsevier Inc.

Li, C.-S.R., Yan, P., Sinha, R., Lee, T.-W., 2008. Subcortical processes of motor response inhibition during a stop signal task. NeuroImage 41, 1352-63. doi:10.1016/j.neuroimage.2008.04.023

Liao, D.-L., Huang, C.-Y., Hu, S., Fang, S.-C., Wu, C.-S., Chen, W.-T., Lee, T.S.-H., Chen, P.C., Li, C.-S.R., 2014. Cognitive control in opioid dependence and methadone maintenance treatment. PloS one 9, 1-7. doi:10.1371/journal.pone.0094589

Liston, C., Matalon, S., Hare, T. a., Davidson, M.C., Casey, B.J., 2006. Anterior Cingulate and Posterior Parietal Cortices Are Sensitive to Dissociable Forms of Conflict in a TaskSwitching Paradigm. Neuron 50, 643-653. doi:10.1016/j.neuron.2006.04.015

Logan, G.D., Cowan, W.B., Davis, K. a, 1984. On the ability to inhibit simple and choice reaction time responses: a model and a method. Journal of experimental psychology Human perception and performance 10, 276-291. doi:10.1037/0096-1523.10.2.276

Luks, T.L., Simpson, G. V., Feiwell, R.J., Miller, W.L., 2002. Evidence for Anterior Cingulate Cortex Involvement in Monitoring Preparatory Attentional Set. NeuroImage 17, 792-802. doi:10.1006/nimg.2002.1210

Menon, V., Uddin, L.Q., 2010. Saliency, switching, attention and control: a network model of insula function. Brain Structure and Function 214, 655-667. doi:10.1007/s00429-010-02620

Mostofsky, S., Simmonds, D., 2008. Response inhibition and response selection: two sides of the same coin. Journal of Cognitive Neuroscience 20, 751-761. 
Muhle-Karbe, P.S., De Baene, W., Brass, M., 2014. Do tasks matter in task switching? Dissociating domain-general from context-specific brain activity. NeuroImage 99, 332-341. doi:10.1016/j.neuroimage.2014.05.058

Nachev, P., Kennard, C., Husain, M., 2008. Functional role of the supplementary and presupplementary motor areas. Nature reviews Neuroscience 9, 856-869. doi:10.1038/nrn2478

Neubert, F.-X., Mars, R.B., Buch, E.R., Olivier, E., Rushworth, M.F.S., 2010. Cortical and subcortical interactions during action reprogramming and their related white matter pathways. Proceedings of the National Academy of Sciences of the United States of America 107, 13240-13245. doi:10.1073/pnas.1000674107

Nichols, T., Brett, M., Andersson, J., Wager, T., Poline, J.B., 2005. Valid conjunction inference with the minimum statistic. NeuroImage 25, 653-660. doi:10.1016/j.neuroimage.2004.12.005

Obeso, I., Robles, N., Marrón, E.M., Redolar-Ripoll, D., 2013. Dissociating the Role of the preSMA in Response Inhibition and Switching: A Combined Online and Offline TMS Approach. Frontiers in human neuroscience 7, 1-9. doi:10.3389/fnhum.2013.00150

Penny, W., Holmes, A., 2003. Random-Effects Analysis, in: Human Brain Function. pp. 843850. doi:10.1016/B978-012264841-0/50044-5

Rae, C.L., Hughes, L.E., Weaver, C., Anderson, M.C., Rowe, J.B., 2014. Selection and stopping in voluntary action: A meta-analysis and combined fMRI study. NeuroImage 86, 381-391. doi:10.1016/j.neuroimage.2013.10.012

Roberts, R.E., Husain, M., 2015. A dissociation between stopping and switching actions following a lesion of the pre-supplementary motor area. Cortex 63, 184-195. doi:10.1016/j.cortex.2014.08.004

Rushworth, M.F., Paus, T., Sipila, P.K., 2001. Attention systems and the organization of the human parietal cortex. The journal of neuroscience 21, 5262-5271. doi:21/14/5262 [pii]

Sharp, D.J., Bonnelle, V., De Boissezon, X., Beckmann, C.F., James, S.G., Patel, M.C., Mehta, M. a, 2010. Distinct frontal systems for response inhibition, attentional capture, and error processing. Proceedings of the National Academy of Sciences of the United States of America 107, 6106-6111. doi:10.1073/pnas.1000175107

Stuphorn, V., Schall, J.D., 2006. Executive control of countermanding saccades by the supplementary eye field. Nature neuroscience 9, 925-31. doi:10.1038/nn1714

Swick, D., Ashley, V., Turken, U., 2011. Are the neural correlates of stopping and not going identical? Quantitative meta-analysis of two response inhibition tasks. NeuroImage 56, 1655-1665. doi:10.1016/j.neuroimage.2011.02.070

Uddin, L.Q., 2015. Salience processing and insular cortical function and dysfunction. Nature Reviews Neuroscience 16, 55-61. doi:10.1038/nrn3857

Verbruggen, F., Logan, G.D., 2008. Response inhibition in the stop-signal paradigm. Trends in Cognitive Sciences 12, 418-424. doi:10.1016/j.tics.2008.07.005

Vink, M., Kaldewaij, R., Zandbelt, B.B., Pas, P., du Plessis, S., 2015. The role of stop-signal probability and expectation in proactive inhibition. European Journal of Neuroscience 41, 1086-1094. doi:10.1111/ejn.12879

Walther, S., Friederich, H.-C., Stippich, C., Weisbrod, M., Kaiser, S., 2011. Response inhibition or salience detection in the right ventrolateral prefrontal cortex? Neuroreport 22, 778-782. doi:10.1097/WNR.0b013e32834af670

Watanabe, T., Hanajima, R., Shirota, Y., Tsutsumi, R., Shimizu, T., Hayashi, T., Terao, Y., Ugawa, Y., Katsura, M., Kunimatsu, A., Ohtomo, K., Hirose, S., Miyashita, Y., Konishi, S., 
2015. Effects of rTMS of Pre-Supplementary Motor Area on Fronto Basal Ganglia Network Activity during Stop-Signal Task. The Journal of Neuroscience 35, 4813-4823. doi:10.1523/JNEUROSCI.3761-14.2015

Wessel, J.R., Aron, a. R., 2013. Unexpected Events Induce Motor Slowing via a Brain Mechanism for Action-Stopping with Global Suppressive Effects. The Journal of Neuroscience 33, 18481-18491. doi:10.1523/JNEUROSCI.3456-13.2013

Wijeakumar, S., Magnotta, V. a., Buss, A.T., Ambrose, J.P., Wifall, T. a., Hazeltine, E., Spencer, J.P., 2015. Response control networks are selectively modulated by attention to rare events and memory load regardless of the need for inhibition. NeuroImage 120, 331-344. doi:10.1016/j.neuroimage.2015.07.026

Winkler, A.D., Hu, S., Li, C.S.R., 2013. The influence of risky and conservative mental sets on cerebral activations of cognitive control. International Journal of Psychophysiology 87, 254-261. doi:10.1016/j.ijpsycho.2012.08.010

Xu, B., Levy, S., Butman, J., Pham, D., Cohen, L.G., Sandrini, M., 2015. Effect of foreknowledge on neural activity of primary "go" responses relates to response stopping and switching. Frontiers in Human Neuroscience 9, 1-12. doi:10.3389/fnhum.2015.00034

Zandbelt, B.B., Bloemendaal, M., Neggers, S.F.W., Kahn, R.S., Vink, M., 2013. Expectations and violations: Delineating the neural network of proactive inhibitory control. Human Brain Mapping 34, 2015-2024. doi:10.1002/hbm.22047

Zandbelt, B.B., Vink, M., 2010. On the role of the striatum in response inhibition. PLoS ONE 5, 1-11. doi:10.1371/journal.pone.0013848

Zar, J.H., 2010. Biostatistical Analysis, Prentice Hall New Jersey USA. doi:10.1037/0012764

Zhang, S., Tsai, S.-J., Hu, S., Xu, J., Chao, H.H., Calhoun, V.D., Li, C.-S.R., 2015. Independent component analysis of functional networks for response inhibition: Inter-subject variation in stop signal reaction time. Human Brain Mapping 26, 3289-3302. doi:10.1002/hbm.22819 
Table 1. Summary of behavioral performance. GoRT = Go Trial reaction time, SERT = stop error reaction time, SSRT = stop signal reaction time, PES = post-error slowing, FP = foreperiod; values are mean \pm standard deviation; $t$ and $p$ values are based on a paired sample $t$ test $(n=16)$.

\begin{tabular}{ccccc}
\hline & SST25 & SST75 & t & p \\
\hline Median GoRT (ms) & $597 \pm 88$ & $787 \pm 137$ & -6.037 & $\mathbf{0 . 0 0 0}$ \\
\hline Median SERT (ms) & $514 \pm 75$ & $696 \pm 134$ & -6.570 & $\mathbf{0 . 0 0 0}$ \\
\hline GoRT - SERT & $94 \pm 32$ & $109 \pm 47$ & -0.964 & 0.350 \\
\hline \% Go Success & $98 \pm 2$ & $89 \pm 11$ & 3.263 & $\mathbf{0 . 0 0 5}$ \\
\hline \% Stop Success & $54 \pm 3$ & $55 \pm 5$ & -1.312 & 0.209 \\
\hline SSRT (ms) & $226 \pm 41$ & $204 \pm 44$ & 1.706 & 0.109 \\
\hline PES (Effect Size) & $1.69 \pm 1.83$ & $1.07 \pm 1.24$ & 1.327 & 0.205 \\
\hline
\end{tabular}


Table 2. Brain regions showing greater activation during SST25 than SST75 in the contrast of stop success (SS) > go success (GS). Thus, these regions showed stronger activation to stopping when stop trials were infrequent, compared to when stopping was the routine. All clusters with peak activities $8 \mathrm{~mm}$ apart are shown.

\begin{tabular}{|c|c|c|c|c|c|}
\hline & & & $\begin{array}{r}\mathrm{MN} \\
\text { oordi } \\
\text { (mn }\end{array}$ & & \\
\hline $\begin{array}{c}\text { Cluster } \\
\text { Size }\left(\mathrm{mm}^{3}\right)\end{array}$ & z-score & X & $\mathrm{Y}$ & Z & Identified Region \\
\hline 20601 & 4.94 & -33 & 20 & 4 & L Insula/Putamen/IFC/Thalamus \\
\hline & 4.7 & -42 & 14 & 4 & \\
\hline & 3.89 & -54 & 8 & 13 & \\
\hline 69552 & 4.93 & 36 & 20 & -8 & R Insula/Putamen/IFC/Thalamus \\
\hline & 3.9 & 48 & 38 & 25 & \\
\hline & 3.9 & 9 & -16 & -11 & \\
\hline 17820 & 4.55 & -54 & -37 & 37 & L Supramarginal Gyrus \\
\hline & 4.21 & -60 & -40 & 46 & \\
\hline & 3.27 & -45 & -49 & 55 & \\
\hline 36018 & 4.41 & 60 & -40 & 40 & R Inferior Parietal Lobule \\
\hline & 4.23 & 63 & -34 & 22 & \\
\hline & 3.79 & 30 & -55 & 40 & \\
\hline 21978 & 4.1 & 6 & 32 & 34 & dACC/preSMA \\
\hline & 3.88 & 6 & 14 & 46 & \\
\hline & 3.75 & -6 & 17 & 43 & \\
\hline 6156 & 3.47 & -54 & -58 & -2 & L Middle Temporal Gyrus \\
\hline & 3.45 & -42 & -55 & -11 & \\
\hline & 2.79 & -63 & -55 & 13 & \\
\hline 1890 & 3.34 & 54 & -22 & -8 & R Middle Temporal Gyrus \\
\hline 1863 & 3.3 & -24 & -70 & -44 & L Cerebellum \\
\hline & 2.71 & -33 & -67 & -44 & \\
\hline & 2.68 & -18 & -61 & -32 & \\
\hline
\end{tabular}




\begin{tabular}{cccccc}
\hline 945 & 3.25 & 0 & -22 & -26 & Midbrain \\
1215 & 3.21 & 51 & -49 & -11 & R Inferior Temporal Gyrus \\
1080 & 3.08 & 0 & -58 & -32 & Cerebellar Vermis \\
\hline
\end{tabular}

Note: R: Right; L: Left; IFC: Inferior Frontal Cortex; dACC: dorsal Anterior Cingulate Cortex:

pre-SMA: pre-Supplementary Motor Area 
Table 3. Brain regions showing a significant correlation between the contrast value of stop success (SS) > go success (GS) and stop-signal reaction time (SSRT). All clusters with peak activities $8 \mathrm{~mm}$ apart are shown.

\begin{tabular}{cccccccc}
\hline & \multicolumn{5}{c}{$\begin{array}{c}\text { MNI } \\
\text { Coordinates } \\
(\mathrm{mm})\end{array}$} \\
\hline $\begin{array}{cccccccc}\text { Experimental } \\
\text { Condition }\end{array}$ & Cluster & Size (mm $\left.{ }^{3}\right)$ & z-score & X & Y & Z & Identified Region \\
\hline SST25 & - & - & - & - & - & (No Significant Results) \\
SST75 & 1350 & 3.14 & -9 & 23 & 46 & dACC \\
& & 2.95 & 9 & 26 & 37 & Pre-SMA \\
& 3051 & 3.78 & 12 & 14 & 58 & \\
& & 3.29 & 15 & 11 & 34 & Thalamus/Striatum \\
& 5751 & 3.68 & -6 & -19 & -2 & \\
& & 3.4 & -9 & -22 & 13 & \\
& & 3.28 & 6 & -13 & 1 & \\
\hline
\end{tabular}


Figure 1. a) Stop-signal task (SST) design. All participants performed two versions of the task: SST25 (25\% stop trials) and SST75 (75\% stop trials). b) The contrast SS > GS measures both response inhibition and salience detection in SST25, but only inhibition in SST75, where stop trials predominate. $\mathrm{SS}=$ stop success, GS = go success, SSD = stop-signal delay. A conjunction of SS > GS between SST25 and SST75 identifies correlates of “stopping," whereas a conjunction of SS > GS in SST25 and GS > SS in SST75 identifies correlates of saliency detection.

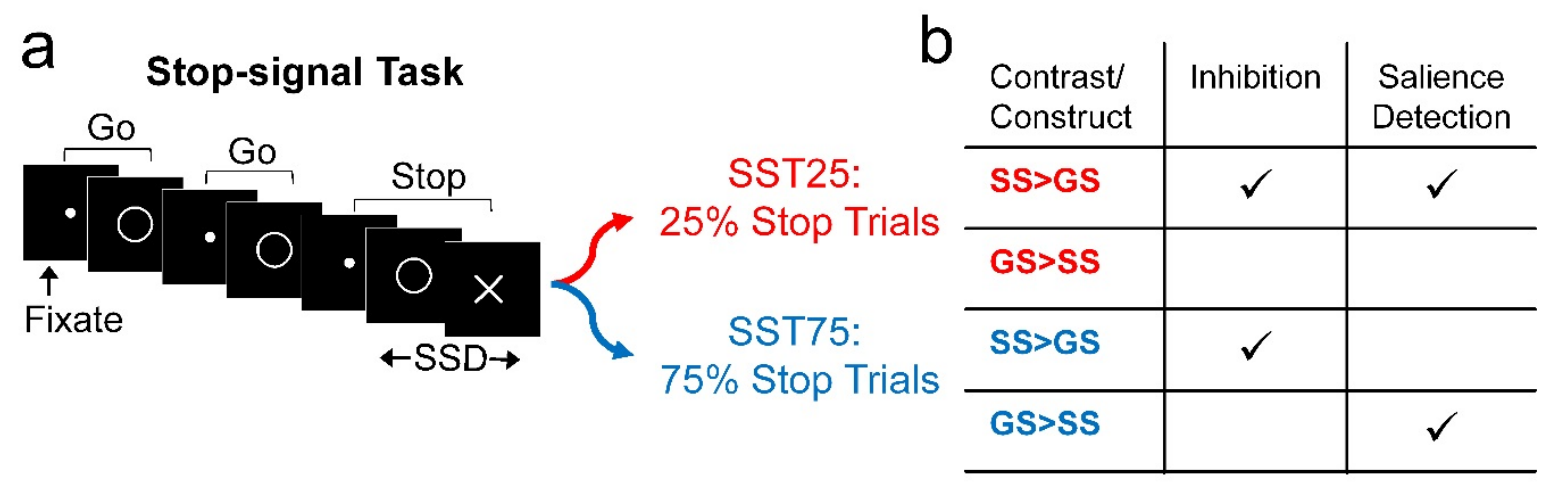


Figure 2. a) Inhibitory functions for the SST25 (red) and SST75 (blue). Dotted lines represent individuals, and bolded solid line represents the group average. b) Voxelwise one-sample t-tests for the contrast stop success (SS) > go success (GS). c) Voxelwise one-sample t-tests for the contrast GS > SS. Overlapping voxels between the two conditions are shown in purple. d) voxelwise paired-sample t test: SST25 > SST 75 (SS > GS).
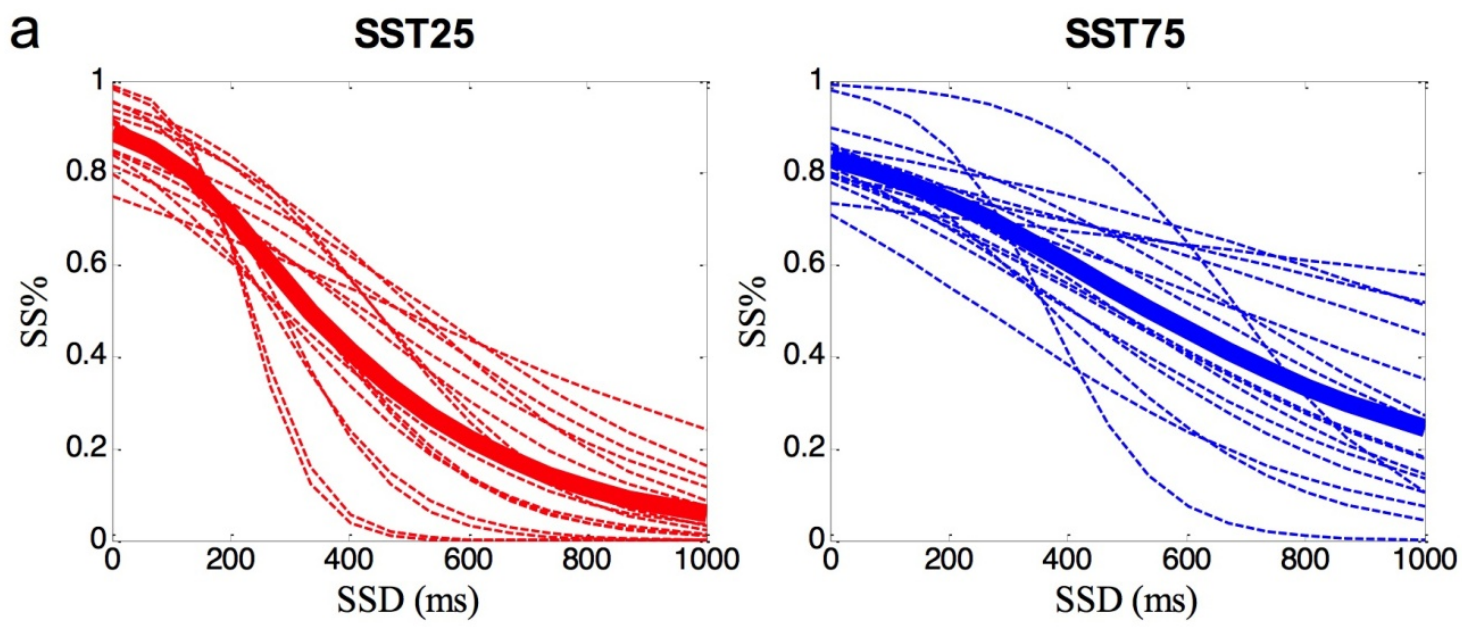

b
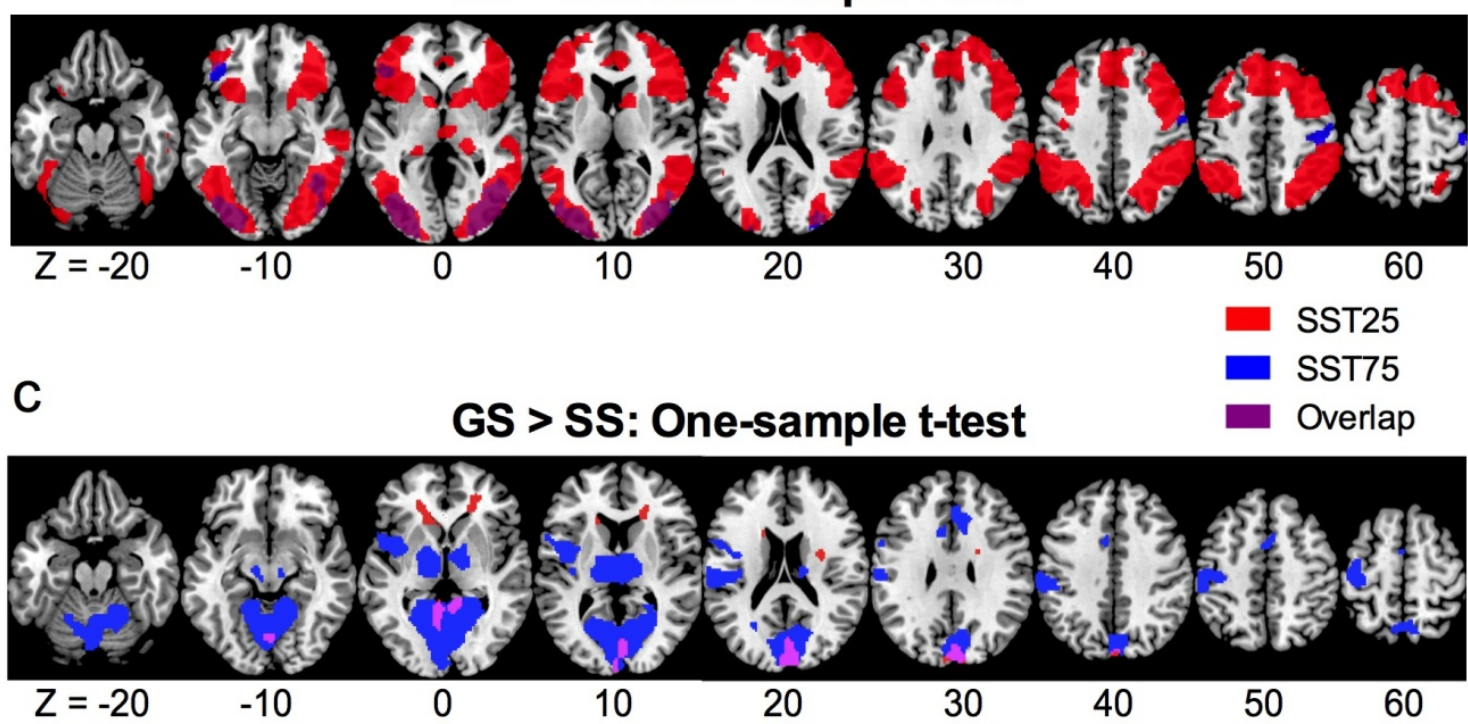

d

SS > GS (SST25 > SST75)

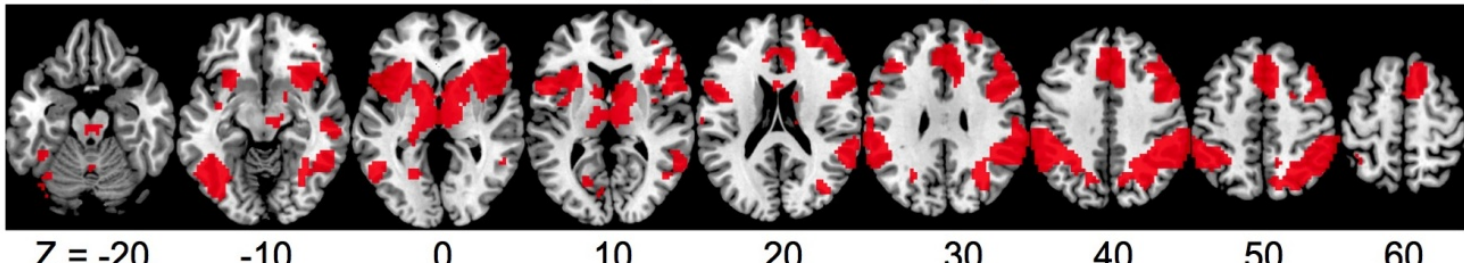

$Z=-20 \quad-10$

10

20

30

40

50

60 
Figure 3. a) Conjunction analysis on "stopping" (SS > GS) of SST25 and SST75 showed clusters in bilateral visual cortices. b) The activity of visual cortical cluster did not correlate with SSRT in SST25 or SST75. c) Conjunction analysis on the infrequent trial type in each condition (SS > GS in SST25 and GS > SS in SST75) revealed a cluster in the dorsal anterior cingulate cortex (dACC), suggesting a role for dACC in saliency detection; d) The activity of this cluster during SS > GS is negatively correlated with SSRT in SST75 but not SST25.

a "Stop" Conjunction: SS>GS: SST25 \& SS>GS: SST75

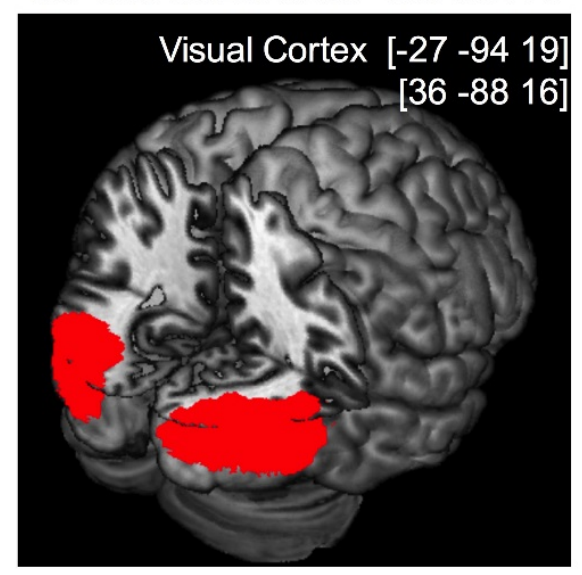

b

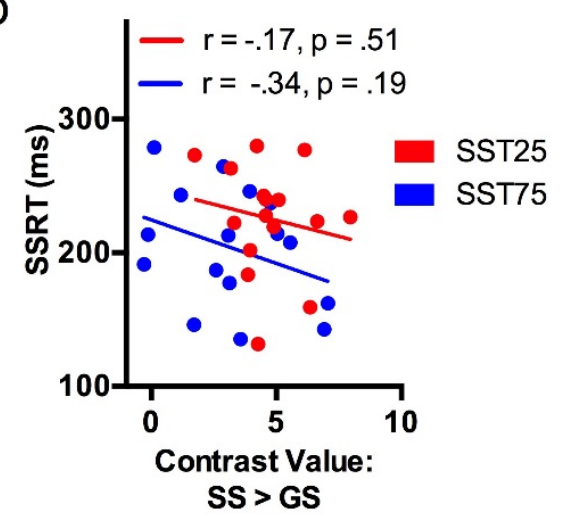

C "Saliency" Conjunction: SS>GS: SST25 \& GS>SS: SST75

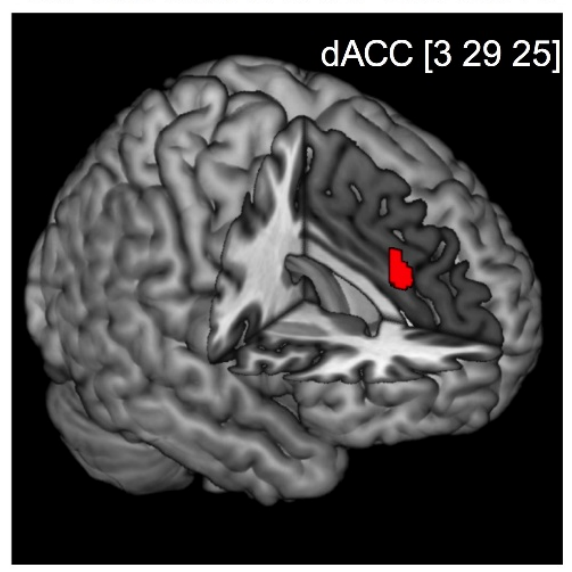

d

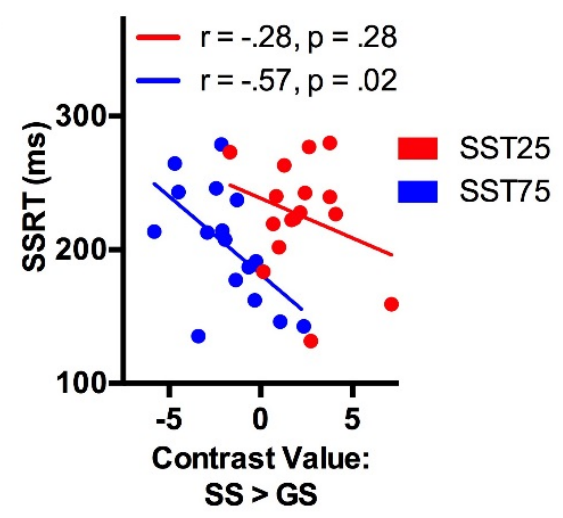


Figure 4. a) Regions of interest analyses identified clusters that showed a negative correlation to SSRT (green) in SST75 in areas with greater activations to SS > GS in SST25 as compared to SST75; no voxels showed a positive correlation to SSRT in SST75 or any correlation to SSRT in SST25. b) Bar plot of contrast values for each cluster (error bar = standard error of the mean). c) Scatterplots for the three clusters showing a significant negative correlation between SS $>$ GS contrast values and SSRT in SST75. SS = stop success, GS = go success, SSRT = stop-signal reaction time, $\mathrm{dACC}=$ dorsal anterior cingulate cortex, pre-SMA = pre-supplementary motor area; Thal $=$ thalamus. $\dagger, \mathrm{p}<.10 ; *, \mathrm{p}<.05 ; * *, \mathrm{p}<.01$

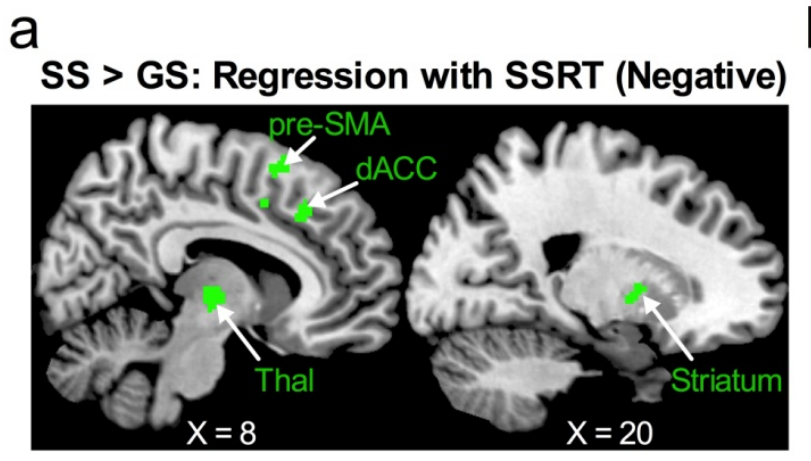

C

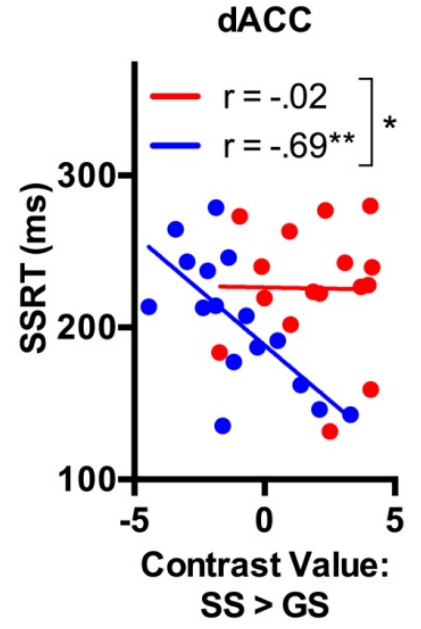

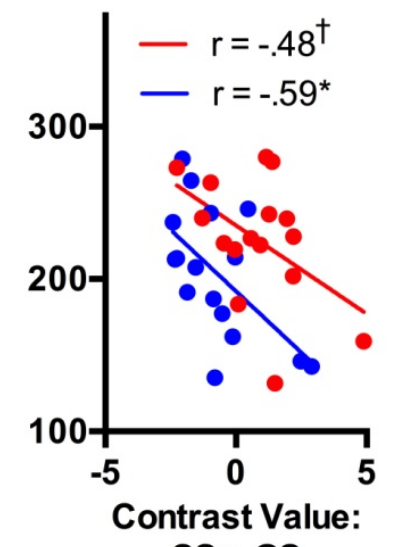

SS > GS

\section{b}

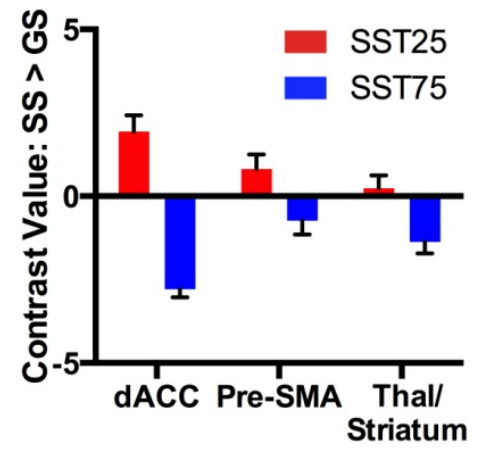

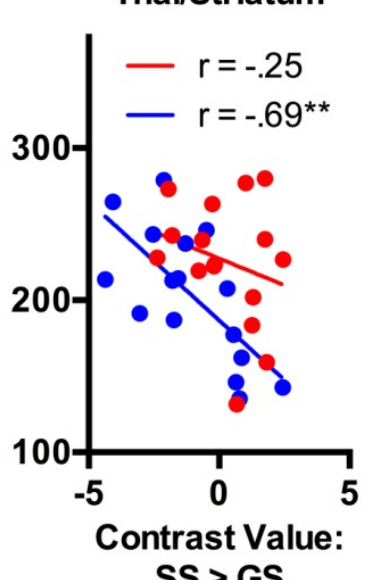

SS > GS 